\title{
Can mammalian mothers influence the sex of their offspring peri-conceptually?
}

\author{
Valerie J Grant ${ }^{1}$ and Lawrence W Chamley ${ }^{2,3}$ \\ ${ }^{1}$ Department of Psychological Medicine and ${ }^{2}$ Department of Obstetrics and Gynaecology, Faculty of Medical and \\ Health Sciences, University of Auckland, Private Bag 92019, Auckland 1142, New Zealand and ${ }^{3}$ Fertility Plus, \\ Green Lane Clinical Centre, Auckland 1142, New Zealand
}

Correspondence should be addressed to V J Grant; Email: vj.grant@auckland.ac.nz

\begin{abstract}
Although controversial, growing evidence from evolutionary biology suggests that the mammalian mother may have a role in influencing the sex of her offspring. However, there is competing information on the molecular mechanisms by which such influence could be manifested. The new initiatives are based on hypotheses from evolutionary biology: the 'good condition' hypothesis, which suggests that post conception, higher levels of maternal glucose may differentially promote the development of male embryos; and the 'maternal dominance' hypothesis, which proposes that before conception, higher follicular testosterone may influence the development of the ovum so that it emerges already adapted to receive an X- or a Y-chromosome-bearing spermatozoon. Now, it seems these hypothesised mechanisms could be operating in synchrony, each complementing and reinforcing the other. On the other hand, there are continuing problems in identifying a precise sequence of mechanisms as evidenced from research in sperm-sorting. Research on high-fat diets and the sex ratio in polytocous species may indicate important differences in proximate mechanisms for sex allocation between polytocous and monotocous mammals.
\end{abstract}

Reproduction (2010) 140 425-433

\section{Introduction}

A growing body of evidence suggests there must be some form of adaptive regulation of mammalian sex ratios. Given that almost all other non-mammalian taxa have efficient adaptive control of the sex ratio of their offspring, and given that such control brings important evolutionary benefits, it seems likely that some kind of mechanism would also be present in mammals. In addition, it would make sense if this adaptive influence was at least partially under maternal control, given the mammalian mother's disproportionate commitment to the production of the offspring. Following the logic of this argument and using the theoretical framework provided by Trivers \& Willard (1973), evolutionary biologists have demonstrated strong (albeit often seemingly contradictory) links between maternal characteristics and statistically significant variations in offspring secondary (birth) sex ratios (Cameron 2004, Sheldon \& West 2004, Grant 2007). Thus, results from many studies in evolutionary biology suggest that even if current hypotheses are somewhat rudimentary, a proximate mechanism for adaptive sex allocation in mammals must surely exist. On the other hand, this thinking is in direct contradiction to the chance theory of sex allocation, which holds that the only influence on the sex of offspring is whether the fertilising sperm carries an $X$ - or a Y-chromosome.

As a result of their findings on atypical secondary sex ratios, evolutionary biologists have offered three theoretically derived clues to those searching for a mechanism. First, it is likely that the mammalian mother will have a role in the allocation of the sex of her offspring. Secondly, it is likely that in a highly conserved system of sex allocation in sexually reproducing animals, the most efficient and least costly time for such a modification to take place would be at, or near, conception. And thirdly, any such system of sex allocation would need to be able to respond to environmental stressors in a way that was evolutionarily advantageous.

Two main research streams have addressed the problems. One is based on the association between maternal dominance and offspring sex ratio (the maternal dominance hypothesis; Grant 1996), and the other between good condition in the mother and the subsequent sex of her offspring (Trivers \& Willard 1973). The Trivers and Willard hypothesis suggested that mammalian parents would gain an evolutionary (fitness) advantage if they could manipulate the sex ratio of their 
offspring. They argued that a mother in good condition would gain maximal evolutionary advantage by producing a son, who, assuming he inherited her condition, would out-reproduce a sister in similar condition. Partly because the difficulties in defining and measuring good condition still remain unresolved (Wilson \& Nussey 2010), maternal dominance, underpinned by testosterone, came to be seen by some researchers not only as a proxy for good condition, but possibly as the key component, overriding environmentally induced changes in condition.

The main factor in resolving the apparently conflicting results appeared to be whether or not the mothers were living under conditions of chronic stress, e.g. food shortages (Verme 1969), population density increases (Kruuk et al. 1999) insufficient den or nesting sites (Clark 1978). Under such conditions, the atypical sex ratios were reversed, i.e. the mothers in poor condition had significantly more male offspring. This reversal may be accounted for by the fact that in females, testosterone (perhaps because in females, it arises in peripheral tissues under the control of the adrenal glands - also associated with the stress response) rises under conditions of chronic stress (Christiansen 1998). Stressful times also increase differential male vulnerability. Conceiving more males may offset this loss and thus be an integral part of frequency-dependent sex selection, which works to ensure that there are equal numbers of males and females at the time of reproductive maturity (see Grant (2007) and (2009) for further details).

In the search for a proximate molecular mechanism that would fit the evolutionary findings, proponents of each hypothesis pursued a putative physiological correlate in the mother, predicted to have the potential to influence sex allocation. Recognising that testosterone underpins a tendency to dominant behaviour (Bouissou 1978, Christiansen 1998, Grant \& France 2001), researchers who favoured the maternal dominance hypothesis sought an association between female testosterone and offspring sex ratio (Grant \& Irwin 2005, Grant et al. 2008, Helle et al. 2008, Shargal et al. 2008), while those opting for the good condition hypothesis sought links in the area of diet (Rosenfeld et al. 2003, Rosenfeld \& Roberts 2004, Cameron et al. 2008, Mathews et al. 2008), and, more explicitly, unsaturated fats (Alexenko et al. 2007), polyunsaturated fatty acids (Fountain et al. 2008, Green et al. 2008) and glucose (Kimura et al. 2005).

Both streams of research have built on earlier work by reproductive biologists. There have been relevant studies of pre-conceptual maternal diet, investigating variations in both quantity (Verme 1969) and quality of diet (Stolkowski \& Lorrain 1980) for an effect on the sex ratio. There have also been studies of the timing of insemination (Guerrero 1974, France et al. 1984, Weinberg et al. 1995), as well as maternal influences on the uterine environment and sex-selective embryo loss. It is becoming clear that both genetic and environmental influences on the sex of the offspring in mammals are relevant to both prenatal (Navara \& Nelson 2009) and peri-conceptual environments.

\section{Evidence supporting the influence of maternal testosterone on offspring sex ratio}

Independently, four research teams (Grant \& Irwin 2005, Grant et al. 2008, Helle et al. 2008, Shargal et al. 2008, Garcia-Herreros et al. 2010) have investigated the hypothesised relationship between pre-conceptual maternal testosterone and the subsequent sex of the offspring. In one study (Shargal et al. 2008), the dominant females in a small captive herd of Nubian ibexes were found to have higher faecal testosterone and significantly more male offspring than subordinate females who had lower faecal testosterone and more female offspring. In the second, much larger study (Helle et al. 2008), both glucose and testosterone were measured using serum samples from female field voles, taken a few days before conception. This study showed no effect of paternal testosterone or of maternal body condition, but higher levels of both glucose and testosterone in the mothers were significantly associated with higher offspring sex ratios (more males than females). Although this study demonstrated an association between high maternal circulating testosterone level and male-biased litter sex ratio, and was the first to do so in a non-domestic mammal, the authors noted that 'the exact mechanisms responsible for this association...remain unknown.' (Helle et al. 2008).

The third and fourth studies (Grant \& Irwin 2005, Grant et al. 2008) may, however, offer a clue about mechanisms. In these studies, we focused on follicular rather than serum or faecal testosterone. In the female's follicular fluid testosterone occurs at much higher concentrations than it does in blood (in humans, 10 000-30 000 times more), and there is wide variation both between and within individuals over time. These facts provided the basis for the hypothesis that an ovum could emerge each oestrus or menstrual cycle already adapted to preferentially receive an X- or a Y-chromosome-bearing spermatozoon depending on the amount of testosterone it was exposed to at a crucial time during its development within the follicle. This hypothesised mechanism had the additional theoretical advantage of being the least costly mechanism for sex allocation of any so far suggested.

The two studies had a similar structure. As described elsewhere (Grant \& Irwin 2005, Grant et al. 2008), ova were removed from abattoir-derived bovine follicles. The follicular fluid in which the ovum had developed was collected and labelled to match the ovum, so that each egg could be traced back to the follicular fluid from 
which it originated. After IVF, the sex of each embryo was determined by PCR, and the matched samples of follicular fluid were analysed for testosterone. Findings from the first study (Grant \& Irwin 2005) suggested that the high level of testosterone had to be present earlier in the antral phase of development than first thought. In the second, larger, study of 171 fertilised oocytes (Grant et al. 2008), we found that ova that had developed in follicular fluid with high levels of testosterone were subsequently significantly more likely to be fertilised by Y-chromosome-bearing spermatozoa, particularly if the high testosterone levels were not simply the result of poor aromatisation of the testosterone to oestradiol $\left(\mathrm{OE}_{2}\right)$. These results suggested that there may be a critical time in the development of the zona pellucida (ZP) during which its molecular composition may be subtly influenced by the high levels of follicular testosterone rendering the oocyte more (or less) susceptible to fertilisation by a Y-bearing spermatozoon.

The most recent study to investigate the relationship between maternal testosterone and subsequent offspring sex was also relatively large and examined 140 fertilised bovine oocytes, using methodology which closely replicated that of Grant et al. (2008). In that study, Garcia-Herreros et al. (2010) demonstrated that median testosterone levels were higher in follicles that resulted in subsequently male embryos $(32.12 \mathrm{ng} / \mathrm{ml})$ than subsequently female embryos $(23.98 \mathrm{ng} / \mathrm{ml})$. This difference approached, but was not statistically significant ( $P=0.06$; Garcia-Herreros et al. 2010). Since the methodology of Garcia-Herreros et al. (2010) is so similar to that of Grant et al. (2008), it is possible to combine the summary data from the two studies. The data combined from both studies show that there were 33 subsequently male embryos and 13 subsequently female embryos that were fertilised from follicles with testosterone levels $>300 \mathrm{nM}$. This demonstrates a significant $\left(\chi^{2} P=0.003\right)$ bias for fertilisation of oocytes developing in high testosterone to be fertilised by Y-chromosome-bearing spermatozoa. For a summary of the findings of these two studies, see Table 1.

Although Garcia-Herreros et al. (2010) did not achieve a significant effect in the conventional sense
( $P=0.06$ for a test of the difference between medians), nevertheless they did successfully replicate the results of Grant et al. (2008) (Table 1). Garcia-Herreros et al.'s (2010) findings satisfy Killeen's (2005) criteria for a successful replication, namely that an equipotent experiment obtains an effect of the same sign as that of the original experiment.

\section{Evidence supporting the influence of maternal condition on offspring sex ratio}

In 1973, Trivers \& Willard proposed that mothers in good condition could maximise fitness by producing male offspring (since a male in good condition could outreproduce a sister in similar condition) thus providing a plausible context for studying maternal body condition and, more recently, the dietary pathway towards achieving good condition. In the decades that followed, evolutionary biologists who found that superior maternal body condition was significantly associated with higher secondary sex ratios (more male offspring at birth) frequently interpreted their findings as evidence supporting the Trivers and Willard hypothesis (for a review see Cameron (2004)).

More recently, experimental interventions have demonstrated a significant effect of pre-conceptual maternal diet on the sex ratio (Alexenko et al. 2007, Fountain et al. 2008, Green et al. 2008). Female mice fed a very high saturated fat diet had significantly more male offspring than either control or restricted fat diet females. For example, in Alexenko et al.'s (2007) study, 244 mice fed a very high saturated fat diet had $60 \%$ male offspring compared to 274 controls that had $48 \%$ male offspring $(P<0.0001)$. Furthermore, since males fed similar diets had neither more Y-sperm nor sired more sons than daughters, the authors concluded that the dietary effects were 'manifested exclusively through the female' (Alexenko et al. 2007). Ewes fed a diet enriched with polyunsaturated fatty acids also had significantly more male offspring (Green et al. 2008), and a large study of human pre-conceptual diet appeared to demonstrate a similar effect (Mathews et al. 2008), although an alternative interpretation of these data has recently

Table 1 Comparison of the main characteristics of studies of bovine follicular testosterone by Grant et al. (2008) and Garcia-Herreros et al. (2010).

\begin{tabular}{|c|c|c|c|c|c|c|}
\hline \multirow[b]{2}{*}{ Study } & \multirow[b]{2}{*}{$\mathbf{N}$ (embryos) } & \multirow{2}{*}{$\begin{array}{l}\text { Follicular size: } \\
\text { min-max }(\mathrm{mm})\end{array}$} & \multirow{2}{*}{$\begin{array}{l}\text { Follicular testosterone: } \\
\qquad \min -\max (\mathrm{nM})\end{array}$} & \multicolumn{2}{|c|}{$\begin{array}{l}\text { Median follicular testosterone } \\
\text { of subsequent embryos }(n M)\end{array}$} & \multirow[b]{2}{*}{ Significance $(P)$} \\
\hline & & & & Male & Female & \\
\hline Grant et al. (2008) & 171 & $4-10$ & $11-977$ & 122.60 & 90.75 & $0.032^{\mathrm{a}}$ \\
\hline Garcia-Herreros et al. (2010) & 140 & $2-12$ & 13-893 & 111.36 & 83.13 & $0.06^{\mathrm{b}}$ \\
\hline
\end{tabular}

${ }^{\mathrm{a}}$ Two-tailed. Grant et al. reported a one-tailed value because a directional hypothesis was tested. ${ }^{\mathrm{b}}$ Two-tailed. The $P$ value was reported for the Wilcoxon signed rank test, which is for related samples. We assume that the Wilcoxon rank sum test, which is for independent samples, was intended (see Garcia-Herreros et al. (2010), p 535). 
been offered by Young et al. (2009) on the grounds that multiple testing may result in false positives.

Although researchers have shown that pre-conceptual diets can be related to offspring sex ratio, the proximate (molecular) mechanisms thought to be associated with this effect have largely been thought to act post conception. That is to say, although the nutritionally induced high maternal glucose levels are likely to exist before conception, the researchers suggested that they act post conception by differentially promoting the development of male embryos (Kimura et al. 2005, 2008). These findings in turn are consistent with earlier work by reproductive biologists who found that male embryos developed more quickly than female embryos in both experimental (Mittwoch 1989, 1996, Ray et al. 1995) and clinical settings (Permagent et al. 1994).

Researchers pursuing the good condition hypothesis (Cameron 2004) have now suggested it might not be good condition per se that was the best predictor of offspring sex, but rather a change in condition, mothers whose condition was improving at the time of conception being more likely to bear males (Cameron \& Linklater 2007). Studies with both murine (Cameron et al. 2008) and bovine (Kimura et al. 2005) embryos showed that increasing amounts of glucose in the uterine environment post conception were associated with the preferential development of males. These findings are consistent with earlier work that documented asynchronous development in embryos in several species, with males developing faster than females (Krackow 1995).

\section{A possible sequence of effects}

Thus, taken as a whole, recent work suggests that these two influences (maternal testosterone prior to conception (Grant \& Irwin 2005) and maternal glucose immediately post conception (Kimura et al. 2005) may have a combined effect on the sex ratio in mammals acting in sequence, and possibly in synchrony with one another. Such a system would have its own integrity, based initially on normally distributed female testosterone, with levels above the mean predisposing the female to more dominant behaviour, which in turn has a higher energy requirement (Rosenfeld \& Roberts 2004). At the same time, as higher testosterone levels in follicular fluid may be instrumental in modifying the ovum to preferentially (but not exclusively) receive either an X- or a Y-chromosome-bearing spermatozoon, variations in glucose levels in the uterine environment may be differentially supportive of either a male or a female embryo. Thus, both pre- and post conception, behaviourally and physiologically, the mammalian mother may be specifically adapted at the time of fertilisation to conceive and nourish either one sex or the other.

Furthermore, both hypotheses fulfil the evolutionary requirements. Each proposes a role for the mother and suggests a way in which she could influence the sex of her offspring. Both suggest proximate mechanisms that would minimise the costs of sex allocation, acting either immediately before, or immediately after, fertilisation. And each has the potential to be differentially responsive to changes in the environment. Regarding the good condition hypothesis, when resources are scarce, body condition deteriorates and glucose levels may decline. Regarding the maternal dominance hypothesis, since female testosterone rises in response to environmental stressors (Christiansen 1998), there would be an opportunity for raised or lowered maternal testosterone to influence sex allocation at the exact time such influence was evolutionarily advantageous.

However, in spite of the neat theoretical fit, both hypotheses require both replication and further exploration. A comparatively simple yet potentially persuasive series of observations could be undertaken in either the field or the laboratory, preferably in different species, to assess the validity or otherwise of measuring preconceptual maternal hormones and glucose as an indicator of future sex of offspring. As cited above, five studies (three laboratory and two field studies) have shown that pre-conceptual hormone profiles may provide an indication of subsequent sex of offspring. One of the field studies measured serum testosterone and glucose, and the other study measured faecal testosterone; the laboratory studies measured follicular testosterone. If further studies were undertaken, particular attention might be paid to both the timing of collection within the pre-conceptual cycle and the measurement of both testosterone and $\mathrm{OE}_{2}$ in order to optimise the likelihood of capturing the full extent of the hypothesised hormonal influence.

A recent example of such a study is that by Perret (2005) who used a very small primate, the grey mouse lemur, as the animal model. Urinary oestrogen levels measured prior to oestrus were significantly predictive of offspring sex, with females that later conceived male offspring having significantly less urinary oestrogen. Since the ratio of oestrogen to testosterone in follicular fluid has long been thought to be relevant to successful oocyte development (McNatty et al. 1984), it is possible to speculate that less oestrogen earlier in the follicular phase could be indicative of higher testosterone, and the high levels of oestrogen at the end of the follicular phase could reflect the rapid aromatisation of testosterone towards the end of the antral phase of the oocyte development. However, it would be preferable not to have to make this speculation and, thus, testing for both oestrogen and testosterone early in the follicular phase may offer a relatively non-invasive method for exploring the hypothesised maternal influence on the sex of the offspring prior to conception.

Although the experiments described above offer tantalising evidence that mammalian mothers might be able to influence the sex of their offspring, there remain significant unresolved problems. 


\section{Problem 1: issues of timing and oocyte development in the maternal testosterone hypothesis}

In spite of the theoretical consistency in the current hypotheses, there remain several problems and inconsistencies. The first of these is exemplified in a study of sex-sorted spermatozoa for use in bovine reproduction (Bermejo-Alvarez et al. 2008). The problem is if, as suggested, an ovum is produced each oestrus cycle already adapted to preferentially receive an X- or a Y-chromosome-bearing spermatozoon, then exposure to any number of spermatozoa of the 'wrong sex' would mean that fertilisation was less likely. Thus, as described elsewhere (Grant \& Chamley 2007), the reported low fertility of animals fertilised with sex-sorted (X-chromosome-bearing) sperm was consistent with the hypothesis that only some of the mothers had produced ova adapted to receive $\mathrm{X}$-chromosome-bearing spermatozoa.

In an elegant study, Bermejo-Alvarez et al. (2008) reported, for the first time, fertility rates and sex ratios produced by sex-sorted, sex-sorted and then recombined, and non-sorted spermatozoa. They found no differences in fertility rates from the three types of sexsorted sperm, and concluded that the differences in cleavage and blastocyst development using sorted versus unsorted sperm' were 'not due to the oocyte preferentially selecting sperm of one sex over another' ...but were 'more likely due to sperm damage caused by the sorting procedure'. These findings suggested that oocytes are not pre-adapted to preferentially accept spermatozoa that bear either an X- or a Y-chromosome. Therefore, if mammalian mothers could influence the sex of their offspring, it was unlikely to be via the method of differential selection of an X- or a Y- chromosomebearing spermatozoon at the ZP.

However, if the initial influence of a mother on the sex of her offspring is via an influence of testosterone levels on the developing ZP, then the timing of the recovery of oocytes is likely to be important since testosterone levels and the testosterone/ $\mathrm{OE}_{2}$ ratio change dynamically during follicular development. The extreme complexity of the cellular environment and production of hormones within the follicle have recently been described (Irving-Rodgers et al. 2003, Rodgers \& Irving-Rodgers 2010). Discriminating between antral and basal follicular atresia, the authors emphasised both the rate of follicular development ('on average, a net 19 doublings in the surface area of the follicle from a primordial to an $18 \mathrm{~mm}$ bovine follicle') and the precise timing of the hormone sequences (for example, 'a significant negative relationship between transforming growth factor...in follicular fluid and follicle diameter occurs at $6.5 \mathrm{~mm}$ (before selection), but not at later stages'). Although in the past, high levels of follicular testosterone were thought to indicate approaching folliclar atresia, recent findings suggest a modification of this view that takes into account the precise stage of development of the follicle, as well as the differing characteristics of basal and antral atresia. For example, there was no statistical difference in the levels of testosterone between antral atretic and healthy follicles $<5 \mathrm{~mm}$ in diameter (Irving-Rodgers et al. 2003).

In the most recent studies examining the relationship between follicular testosterone and sex ratios, bovine follicles were selected according to size, diameters ranging from 2 to $8 \mathrm{~mm}$ (Bermejo-Alvarez et al. 2008), from 4 to $10 \mathrm{~mm}$ (Grant et al. 2008) and from 2 to $12 \mathrm{~mm}$ (Garcia-Herreros et al. 2010). But gross measurement of follicle size is likely to be a poor indicator of the stage of follicular development, and it may be necessary to further refine this in future investigations.

In our earlier studies (Grant \& Irwin 2005, Grant et al. 2008) as well as in the more recently published study by Garcia-Herrreros et al. (2010), the distribution of testosterone in follicular fluid was markedly skewed to the right, with high levels of testosterone associated with fertilisation by Y-chromosome-bearing spermatozoa. As noted above, testosterone levels over $300 \mathrm{nmol} / \mathrm{l}$ were strongly predictive of subsequently male embryos, whereas the lower amounts of follicular testosterone did not discriminate between subsequently male and female embryos, and this held true when the $\mathrm{OE}_{2}$ to testosterone ratio was used to restrict our analysis to only those follicles at the same stage of development. Hence, we suggested that it may not simply be the level of testosterone that is important but the timing of the testosterone peak as well as the cause of the peak (i.e. increased testosterone synthesis rather than reduced aromatisation) that is relevant to the conditioning of the $\mathrm{ZP}$ for preferentially accepting an X- or a Y-chromosomebearing spermatozoon.

\section{Problem 2: the ZP in the maternal testosterone hypothesis}

If, as hypothesised, the ZP has a potential gate-keeping role in sex allocation, more information is needed about the form this role could take. Although the functions of the ZP may include responsibility for species specificity and facilitation of the acrosome reaction, Wassarman (2008) wrote 'many important issues related to the participation of the ZP glycoproteins in the fertilisation process remain unresolved or controversial'. At the same time, he noted that such processes are likely to be highly conserved 'during several hundred million years of evolution'. In the absence of a competing hypothesis, it continues to be logical to suggest that the ZP could be the site of a modifiable process involving pre-conceptual sex allocation. Such a modification might for example be a subtle variation in a carbohydrate (sperm-binding ligand) on the ZP proteins.

In the setting of human reproduction, there is some potentially corroborative evidence from clinical studies 
involving ICSI. When the sperm is injected directly into the ovum, it bypasses the $\mathrm{ZP}$, thus also bypassing any putative role it might have in influencing sex allocation. Given equal numbers of $\mathrm{X}$ - and $\mathrm{Y}$-chromosome-bearing spermatozoa are present in the ejaculate (Graffelmann et al. 1999), it might be expected that the resultant birth (secondary) sex ratio would be no different to chance (100 males to 100 females). In fact, there are significantly lower than normal secondary sex ratios (i.e. fewer males) following ICSI. One study found a ratio of 96:100 (Luke et al. 2009) and another 101:100 (Fedder et al. 2007) males:females following ICSI. Although the normal primary (conception) sex ratio is considerably higher (Hassold et al. 1983), the normal secondary (birth) sex ratio in humans is 105 or 106 male births for every 100 female births.

Differential male vulnerability, which operates from conception onwards, has been demonstrated in animals (Forsyth et al. 2004), and extensively documented in humans (Kruger \& Nesse 2004). It is said to account for the fact that more males than females are both conceived and born. In evolutionary terms, it appears that one of the reasons more males are conceived in the first place is to counterbalance differential male vulnerability (Grant 2009). However, when births follow ICSI, this normal conception or primary sex ratio (higher number of males) is bypassed leaving the most likely influence on the conception sex ratio to be the equal numbers of $X$ - and Y-chromosome-bearing spermatozoa. In this setting, therefore, only male vulnerability is left to modify the birth sex ratio. The consequence is that equal numbers of the sexes may well be conceived, but more males will be lost post conception, resulting in fewer males than usual being born (Fedder et al. 2007, Luke et al. 2009). This suggests that in the absence of a potential influence of the ZP during ICSI, random sex allocation does occur in humans, but when the ZP is present (i.e. in normal conceptions), more males are conceived.

\section{Problem 3: seemingly contradictory results in studies of the good condition hypothesis}

The seeming contradictions in studies of the good condition hypothesis are as follows: first, researchers found that female mice fed a diet high in saturated fat produced significantly more male offspring (Rosenfeld et al. 2003). These results were consistent with the Trivers and Willard hypothesis providing experimental evidence in support of the proposition that mothers in good condition would produce more males. Next, researchers showed that it was the high-fat content of the diet, rather than the number of calories, which led to the production of more male offspring (Alexenko et al. 2007). At the same time, it was reported that these changes in diet had led to changes in serum hormones (Whyte et al. 2007), but instead of the high-fat diet being associated with increased serum testosterone around the time of conception (which could have supported both the maternal dominance hypothesis and the good condition hypothesis), it was found instead to be associated with significantly lower serum testosterone. Half a day postcoitus, testosterone was substantially lower in the mice fed the very high-fat diet $(10.5 \pm 3.0 \mathrm{pg} / \mathrm{ml})$ compared with the low-fat group $(32.7 \pm 8.4 \mathrm{pg} / \mathrm{ml})$.

Then, further investigation on the effect of high-fat diets (which involved discriminating between diets high in the omega 3 and omega 6 components of polyunsaturated fatty acids) found that the sex ratios were reversed (Fountain et al. 2008). The mice fed the high-fat diet enriched with omega 6 gave birth to a significantly higher number of female offspring. In all these studies, the diet-induced changes in offspring sex ratio were not trivial. For example, in this study, female mice fed the omega 6-enriched diet had 213 female offspring and 133 male offspring $(P<0.001)$. Neither the omega 3 -enriched nor the control diets had any effect on the birth sex ratio.

From the point of view of synthesising the two main hypotheses, this latest result suggests a degree of compatibility. This is because results from the earlier work (Rosenfeld et al. 2003) in which females fed a highfat diet conceived more male offspring may have provided evidence for the good condition hypothesis as originally suggested, because the particular kind of high-fat diet was not so extreme as to overcome the body's natural homoeostasis; whereas in the later study, the more extreme diet resulted in lowered pre-conceptual serum testosterone which in turn resulted in the conception of significantly fewer male offspring. Thus, further exploration of possible links and/or interaction effects between diets high in polyunsaturated fatty acids and their putative effects on serum testosterone may prove fruitful.

\section{Problem 4: differences between polytocous and monotocous mammals}

An alternative explanation for the seeming contradictions in research on both good condition and maternal testosterone on the secondary sex ratio is that there may be important species differences, in particular, between monotocous and polytocous mammals. It is possible that putative mechanisms providing for adaptive allocation of sex of offspring in monotocous mothers may need to be more focused or specific than they are in polytocous mothers, given that the monotocous mother has only one opportunity to get the sex of her offspring 'right' in any particular breeding season.

Several lines of evidence support this suggestion. First, at the epidemiological level, James (2009) has pointed out that the distribution of the sexes in polytocous mammals is sub-binomial; i.e. mathematically, 'there are too many litters in which the sexes are equally balanced, 
and there are too few unisexual litters' (James 2009). This he says is 'most unusual in nature' and requires an explanation. His suggestion is that the sub-binomial distribution arises because in polytocous mammals, zygotes within a litter are formed across a period of time and hence may be differently influenced by the relevant maternal hormones.

Next, at the level of individual, behavioural and social characteristics, Novakova et al. (2010) in a large study ( $n=4048$ spiny mice newborns) could find no difference in offspring sex ratio on a range of maternal attributes including parity, age in days or litter size; nor in eight social variables including the number of adult males present, founding time of the breeding group or maternal status (as defined by whether or not she was 'first breeding founder'). It should be noted that these authors did not attempt any manipulation of the offspring sex ratio. They did, however, offer reasons why manipulation of the secondary sex ratio may not be necessary in these animals. Spiny mice in the wild, they wrote, 'strictly avoid breeding whenever they perceive the environmental or social conditions as not fully favourable'; and 'there may be reduced variance in body condition and consequently, no reason for maternal manipulation and/ or any other maternal effect on the sex ratio of the progeny'. Both these reasons contribute to the debate about whether there would or would not be evolutionary incentives for polytocous mothers to have an influence on the sex ratio of the offspring in the same way as monotocous mothers.

At the proximate level, there is evidence that in mice, oocytes are not selective towards X- or Y-bearing sperm (Zuccotti et al. 2005). By doubling the sperm concentration, these researchers were able to override natural barriers to polyspermy. Their results showed $X$ - and Y-sperm fertilisations, which are entirely consistent with a mathematically predictable distribution of the sexes on the basis of chance. The work was done with metaphase II oocytes, and whether this stage of development is sufficiently mature to allow an influence on the ZP in mice is unknown. However, as noted above, there is some evidence that the ZP2 in mice functions differently to that in other species (Hinsch et al. 1998). Therefore, a more parsimonious explanation for these results could be that no selection was taking place at the ZP.

Taken together, these multi-level findings (epidemiological, behavioural and physiological) suggest that putative maternal mechanisms influencing sex ratio manipulation in polytocous mammals could be somewhat different from those hypothesised to exist in monotocous mammals.

Furthermore, an example of a different mechanism for a maternal influence on offspring sex ratio in polytocous mammals was demonstrated by Clark et al. (1993) in gerbils, and by Vandenbergh \& Huggett (1994) in mice. Both studies showed that offspring sex ratios were affected by maternal testosterone. In both instances, the higher maternal testosterone was shown to have originated during the female's development in utero. The source of the higher testosterone may have been from the neighbouring male foetuses. Since the females thus androgenised not only produced significantly more male offspring when they had their first litters, but more males throughout their reproductive lives, this effect too warrants further exploration.

More recently, it was found that bovine embryos and calves originating from the right ovary were more likely to be male, while those from the left ovary were more likely to be female (Hylan et al. 2009). At the same time, the highly significant effects on the sex ratio, which were found in naturally bred cattle, were not demonstrated in embryos transplanted after IVF. But as the oocytes used in the IVF programme measured between 2 and $9 \mathrm{~mm}$, the same phenomenon could apply here as in the spermsorting experiments. Unless the oocytes are studied at the appropriate stage of development of the ZP, the ZPs may not demonstrate the ability to influence offspring sex.

\section{Problem 5: do males contribute to adaptive control of the sex ratio in mammals?}

Although the focus of this paper has been on ways in which the mammalian mother might be able to adaptively influence the sex of her offspring, there remains a question about whether or not there may also be a pre-conceptual paternal influence on the sex of the offspring. In polygynous mammals, a male in good condition will out-reproduce, often by a wide margin, all the other males in the group. Provided that the males disperse, thus minimising in-breeding, there should be an adaptive benefit since the highest status male is also likely to be in the best condition. The sperm characteristics of such males have been described in detail, most recently by Malo et al. (2005), and include measures of both quantity and quality (e.g. proportion of motile and morphologically normal spermatozoa). Recently, the same team (Gomendio et al. 2006) showed that semen from 'more fertile' males (defined as impregnating a higher proportion of females) produced a higher number of male offspring. If replicated, this would suggest that males too could have a role in the adaptive control of the sex ratio. On the other hand, the experimenters reported a $50.39 \%$ fertility rate, and the hinds were synchronised in order to control for timing effects of insemination on the sex ratio. But if females are conceived later in the cycle (Weinberg et al. 1995), this manipulation may have countered the conception of female offspring.

\section{Conclusion}

For many years, the dogma has been that the sex of mammals is determined solely by chance, depending on whether an X- or Y-chromosome-bearing spermatozoon fertilises the oocyte. This thinking is now being 
challenged by growing evidence from field, clinical and laboratory studies, which suggests that mammalian mothers may have some influence over the sex of their offspring. What is now required is the experimental confirmation of one or more molecular mechanisms demonstrating the existence (or not) of a testosteroneinduced modification to the ZP (pre conception) in monotocous females and/or a mechanism whereby male, rather than female, zygotes are advantaged by the presence of glucose in the uterine environment. Unless such mechanisms can be convincingly demonstrated, no matter how much behavioural and theoretical evidence accumulates, the hypotheses will remain nothing more than hypotheses. On the other hand, having the ability to modify natural sex selection processes, if they exist, would provide advantages to both livestock industries and mammalian conservation programmes, both good reasons to pursue these studies in addition to their academic interest.

\section{Declaration of interest}

The authors declare that there is no conflict of interest that could be perceived as prejudicing the impartiality of this review.

\section{Funding}

This review did not receive any specific grant from any funding agency in the public, commercial or not-for-profit sector.

\section{Acknowledgements}

We thank R J Irwin for assistance with Table 1 and comments on the statistics. We also thank the anonymous reviewers and members of the Editorial Board for their constructive comments on an earlier version of this paper.

\section{References}

Alexenko AP, Mao J, Ellersieck MR, Davis AM, Whyte JJ, Rosenfeld CS \& Roberts RM 2007 The contrasting effects of ad libitum and restricted feeding of a diet very high in saturated fats on sex ratio and metabolic hormones in mice. Biology of Reproduction 77 599-604. (doi:10.1095/biolreprod.107.062174)

Bermejo-Alvarez P, Rizos D, Rath D, Lonergan P \& Gutierrez-Adan A 2008 Can bovine in vitro-matured oocytes selectively process X- or Y-sorted sperm differentially? Biology of Reproduction 79 594-597. (doi:10.1095/biolreprod.108.070169)

Bouissou MF 1978 Effects of testosterone propionate on dominance relationships in cows. Hormones and Behavior 11 388-400. (doi:10. 1016/0018-506X(78)90039-9)

Cameron EZ 2004 Facultative adjustment of mammalian sex ratios in support of the Trivers-Willard hypothesis: evidence for a mechanism. Proceedings of the Royal Society of London. Series B 271 1723-1728. (doi:10.1098/rspb.2004.2773)

Cameron EZ \& Linklater WL 2007 Extreme sex ratio variation in relation to change in condition around conception. Biology Letters 3 395-397. (doi:10.1098/rsbl.2007.0089)
Cameron EZ, Lemons PR, Bateman PW \& Bennett NC 2008 Experimental alteration of litter sex ratios in a mammal. Proceedings of the Royal Society of London. Series B 275 323-327. (doi:10.1098/ rspb.2007.1401)

Christiansen K 1998 Behavioural correlates of testosterone. In Testosterone: Action, Deficiency, Substitution, pp 107-142. Eds E Nieschlag \& HM Behre. Heidelberg: Springer-Verlag.

Clark AB 1978 Sex ratio and local resource competition in a prosimian primate. Science 201 163-165. (doi:10.1126/science. 201.4351.163)

Clark MM, Karpluk P \& Galef BG 1993 Hormonally mediated inheritance of acquired characteristics in Mongolian gerbils. Nature 364 712. (doi:10.1038/364712a0)

Fedder J, Gabrielsen A, Humaidan P, Erb K, Ernst E \& Loft A 2007 Malformation rate and sex ratio in 412 children conceived with epididymal or testicular sperm. Human Reproduction 22 1080-1085. (doi:10.1093/humrep/del488)

Forsyth DM, Tustin KG, Gaillard J-M \& Loison A 2004 Fetal sex ratio variation in the highly polygynous Himalayan tahr: evidence for differential male mortality. Behavioral Ecology 15 572-578. (doi:10. 1093/beheco/arh063)

Fountain ED, Mao J, Whyte JJ, Mueller KE, Ellersieck MR, Will MJ, Roberts RM, Macdonald R \& Rosenfeld CS 2008 Effects of diets enriched in omega- 3 and omega- 6 polyunsaturated fatty acids and offspring sex-ratio and maternal behaviour in mice. Biology of Reproduction 78 211-217. (doi:10.1095/biolreprod.107.065003)

France JT, Graham FM, Gosling L \& Hair PI 1984 A prospective study of the preselection of the sex of the offspring by timing intercourse relative to ovulation. Fertility and Sterility $\mathbf{4 1}$ 894-900.

Garcia-Herreros M, Bermejo-Alvarez P, Rizos D, Gutierrez-Adan A, Fahey AG \& Lonergan P 2010 Intrafollicular testosterone concentration and sex ratio in individually cultured bovine embryos. Reproduction, Fertility, and Development 22 533-538. (doi:10. 1071/RD09157)

Gomendio M, Malo AF, Soler AJ, Fernandez-Santos MR, Esteso MC, Garcia AJ, Roldan ERS \& Garde J 2006 Male fertility and sex ratio at birth in red deer. Science 314 1445-1447. (doi:10.1126/science. 1133064)

Graffelmann J, Fugger EF, Keyvanfar K \& Schulman JD 1999 Human live birth and sperm sex ratios compared. Human Reproduction 14 2917-2920. (doi:10.1093/humrep/14.11.2917)

Grant VJ 1996 Sex determination and the maternal dominance hypothesis. Human Reproduction 11 2371-2375.

Grant VJ 2007 Could maternal testosterone levels govern mammalian sex ratio deviations? Journal of Theoretical Biology 246 708-719. (doi:10.1016/j.jtbi.2007.02.005)

Grant VJ 2009 Wartime sex ratios: stress, male vulnerability and the interpretation of atypical sex ratio data. Journal of Evolutionary Psychology 4 251-262. (doi:10.1556/JEP.7.2009.4.5)

Grant VJ \& Chamley LW 2007 Sex-sorted sperm and fertility: an alternative view. Biology of Reproduction 76 184-188. (doi:10. 1095/biolreprod.106.056259)

Grant VJ \& France JT 2001 Dominance and testosterone in women. Biological Psychology 58 41-47. (doi:10.1016/S0301-0511(01) 00100-4)

Grant VJ \& Irwin RJ 2005 Follicular fluid steroid levels and subsequent sex of bovine embryos. Journal of Experimental Zoology 303A 1120-1125. (doi:10.1002/jez.a.233)

Grant VJ, Irwin RJ, Standley NT, Shelling AN \& Chamley LW 2008 Sex of bovine embryos may be related to mothers' preovulatory follicular testosterone. Biology of Reproduction 78 812-815. (doi:10.1095/ biolreprod.107.066050)

Green MP, Spate LD, Parks TE, Kimura K, Murphy CN, Williams JE, Kerley MS, Green JA, Keisler DH \& Roberts RM 2008 Nutritional skewing of conceptus sex in sheep: effects of a maternal diet enriched in rumen-protected polyunsaturated fatty acids (PUFA). Reproductive Biology and Endocrinology 621 (doi:10.1186/14777827-6-21). (doi:10.1186/1477-7827-6-21)

Guerrero R 1974 Association of the type and time of insemination within the menstrual cycle with the human sex ratio at birth. New England Journal of Medicine 291 1056-1059. 
Hassold T, Quillen SD \& Yamane JA 1983 Sex ratio in spontaneous abortions. Annals of Human Genetics 47 39-47. (doi:10.1111/ j.1469-1809.1983.tb00968.x)

Helle S, Laaksonen T, Adamsson A, Paranko J \& Huitus O 2008 Female field voles with high testosterone and glucose levels produce malebiased litters. Animal Behaviour 75 1031-1039. (doi:10.1016/ j.anbehav.2007.08.015)

Hinsch E, Hagele W, Bohle R-M, Schill W-B \& Hinsch K-D 1998 Evaluation of ZP2 domains of functional importance with antisera against synthetic ZP2 peptides. Journal of Reproduction and Fertility 114 245-251. (doi:10.1530/jrf.0.1140245)

Hylan D, Giraldo AM, Carter JA, Gentry GT, Bondioli KR \& Godke RA 2009 Sex ratio of bovine embryos and calves originating from the left and right ovaries. Biology of Reproduction 81 933-938. (doi:10. 1095/biolreprod.109.077727)

Irving-Rodgers HF, Krupa M \& Rodgers RJ 2003 Cholesterol side-chain cleavage cytochrome P450 and 3ß-hydroxysteroid dehydrogenase expression and the concentrations of steroid hormones in the follicular fluids of different phenotypes of healthy and atretic bovine ovarian follicles. Biology of Reproduction 69 2022-2028. (doi:10. 1095/biolreprod.103.017442)

James WJ 2009 The variances of the distributions of the combinations of the sexes within mammalian litters: notes to mark the centenary of the problem. Journal of Theoretical Biology 259 264-268. (doi:10. 1016/j.jtbi.2009.03.027)

Killeen PR 2005 An alternative to null hypothesis significance tests. Psychological Science 16 345-353. (doi:10.1111/j.0956-7976. 2005.01538.x)

Kimura K, Spate LD, Green MP \& Roberts RM 2005 Effects of D-glucose concentration, D-fructose, and inhibitors of enzymes of the pentose phosphate pathway on the development and sex ratio of bovine blastocysts. Molecular Reproduction and Development 72 201-207. (doi:10.1002/mrd.20342)

Kimura K, Iwata H \& Thompson JG 2008 The effect of glucosamine concentration on the development and sex ratio of bovine embryos. Animal Reproduction Science 103 228-238. (doi:10.1016/j.anireprosci.2006.12.014)

Krackow S 1995 The developmental asynchrony hypothesis for sex ratio manipulation. Journal of Theoretical Biology 176 273-280. (doi:10.1006/jtbi.1995.0197)

Kruger DJ \& Nesse RM 2004 Sexual selection and the male:female mortality ratio. Evolutionary Psychology 2 66-85.

Kruuk LEB, Clutton-Brock TH, Albon SD, Pemberton JM \& Guinness FE 1999 Population density affects sex ratio variation in red deer. Nature 399 459-461. (doi:10.1038/20917)

Luke B, Brown MB, Grainger DA, Baker VL, Ginsburg E \& Stern JE 2009 The sex ratio of singleton offspring in assisted-conception pregnancies. Fertility and Sterility 92 1579-1585. (doi:10.1016/ j.fertnstert.2008.08.107)

Malo AF, Garde JJ, Soler AJ, Garcia AJ, Gomendio M \& Roldan ERS 2005 Male fertility in natural populations of red deer is determined by sperm velocity and the proportion of normal spermatozoa. Biology of Reproduction 72 822-829. (doi:10.1095/biolreprod.104.036368)

Mathews F, Johnson PJ \& Neil A 2008 You are what your mother eats: evidence for maternal preconception diet influencing foetal sex in humans. Proceedings of the Royal Society of London. Series B 275 1661-1668. (doi:10.1098/rspb.2008.0105)

McNatty KP, Heath DA, Henderson KM, Lun S, Hurst PR, Ellis LM, Montgomery GW, Morrison L \& Thurley DC 1984 Some aspects of thecal and granulosa cell function during follicular development in the bovine ovary. Journal of Reproduction and Fertility 72 39-53. (doi:10.1530/jrf.0.0720039)

Mittwoch U 1989 Sex differentiation in mammals and tempo of growth: probabilities vs. switches. Journal of Theoretical Biology 137 445-455. (doi:10.1016/S0022-5193(89)80039-6)

Mittwoch U 1996 Differential implantation rates and variations in the sex ratio. Human Reproduction 11 8-9.

Navara KJ \& Nelson RJ 2009 Prenatal environmental influences on the production of sex-specific traits in mammals. Seminars in Cell and Developmental Biology 20 313-319. (doi:10.1016/j.semcdb. 2008.12.004)
Novakova M, Vasakova B, Kutalova H, Galestokova K, Prusova K, Smilauer P, Sumbera R \& Frynta D 2010 Secondary sex ratios do not support maternal manipulation: extensive data from laboratory colonies of spiny mice (Muridae: Acomys). Behavioral Ecology and Sociobiology 64 371-379. (doi:10.1007/s00265-009-0853-z)

Permagent E, Fiddler M, Cho N, Johnson D \& Holmgren WJ 1994 Sexual differentiation and preimplantation cell growth. Human Reproduction 9 1730-1732.

Perret M 2005 Relationship between urinary estrogen levels before conception and sex ratio at birth in a primate, the gray mouse lemur. Human Reproduction 20 1504-1510. (doi:10.1093/ humrep/deh802)

Ray PF, Conaghan J, Winston RM \& Handyside AH 1995 Increased number of cells and metabolic activity in male human preimplantation embryos following in vitro fertilization. Journal of Reproduction and Fertility 104 165-171. (doi:10.1530/jrf.0.1040165)

Rodgers RJ \& Irving-Rodgers HF 2010 Morphological classification of bovine ovarian follicles. Reproduction 139 309-318. (doi:10.1530/ REP-09-0177)

Rosenfeld CS \& Roberts RM 2004 Maternal diet and other factors affecting offspring sex ratio: a review. Biology of Reproduction $\mathbf{7 1}$ 1063-1070. (doi:10.1095/biolreprod.104.030890)

Rosenfeld CS, Grimm KM, Livingston KA, Brokman AM, Lamberson WE \& Roberts RM 2003 Striking variation in the sex ratio of pups born to mice according to whether maternal diet is high in fat or carbohydrate. PNAS 100 4628-4632. (doi:10.1073/pnas. 0330808100)

Shargal D, Shore L, Roteri N, Terkel A, Zorovsky Y, Shemesh M \& Steinberger Y 2008 Fecal testosterone is elevated in high ranking female ibexes (Capra nubiana) and associated with increased aggression and a preponderance of male offspring. Theriogenology 69 673-680. (doi:10.1016/j.theriogenology.2007.11.017)

Sheldon BC \& West SA 2004 Maternal dominance, maternal condition, and offspring sex ratio in ungulate mammals. American Naturalist 163 40-54. (doi:10.1086/381003)

Stolkowski J \& Lorrain J 1980 Preconceptional selection of fetal sex. International Journal of Gynaecology and Obstetrics 18 440-443.

Trivers RL \& Willard D 1973 Natural selection of parental ability to vary the sex ratio of offspring. Science 179 90-92. (doi:10.1126/science. 179.4068.90)

Vandenbergh JG \& Huggett CL 1994 Mother's prior intrauterine position affects the sex ratio of her offspring in house mice. PNAS 91 11055-11059. (doi:10.1073/pnas.91.23.11055)

Verme LJ 1969 Reproduction patterns of white-tailed deer related to nutritional plane. Journal of Wildlife Management 33 881-887. (doi:10.2307/3799320)

Wassarman PM 2008 Zona pellucida glycoproteins. Journal of Biological Chemistry 283 24285-24289. (doi:10.1074/jbc. R800027200)

Weinberg CR, Baird DD \& Wilcox AJ 1995 The sex of the baby may be related to the length of the follicular phase in the conception cycle. Human Reproduction 10 304-307.

Whyte JJ, Alexenko AP, Davis AM, Ellersieck MR, Fountain ED \& Rosenfeld CS 2007 Maternal diet composition alters serum steroid and free fatty acid concentrations and vaginal $\mathrm{pH}$ in mice. Journal of Endocrinology 192 75-81. (doi:10.1677/JOE-06-0095)

Wilson AJ \& Nussey DH 2010 What is individual quality? An evolutionary perspective Trends in Ecology \& Evolution 25 207-214. (doi:10.1016/j.tree.2009.10.002)

Young SS, Bang H \& Oktay K 2009 Cereal-induced gender selection? Most likely a multiple testing false positive Proceedings of the Royal Society of London. Series B 276 1211-1212. (doi:10.1098/rspb. 2008.1405)

Zuccotti M, Sebastiano V, Garagna S \& Redi CA 2005 Experimental demonstration that mammalian oocytes are not selective towards $X$ or Y-bearing sperm. Molecular Reproduction and Development $\mathbf{7 1}$ 245-246. (doi:10.1002/mrd.20252)

Received 15 March 2010

First decision 27 April 2010

Accepted 30 June 2010 